\title{
Construction and Evaluation of Cysteine Selective Electrodes for FIA Analysis of Pharmaceuticals
}

\author{
M. Goreti F. Sales, ${ }^{*}$ Annouschka Pille, and \\ Paula C. B. Pai'ga
}

\begin{abstract}
A flow injection analysis (FIA) system comprising a cysteine selective electrode as detection system was developed for determination of this amino acid in pharmaceuticals. Several electrodes were constructed for this purpose, having PVC membranes with different ionic exchangers and mediator solvents. Better working characteristics were attained with membranes comprising $o$-nitrophenyl octyl ether as mediator solvent and a tetraphenylborate based ionic-sensor.
\end{abstract}

Injection of $500 \mathrm{~mL}$ standard solutions into an ionic strength adjuster carrier $\left(3 \times 10^{-3} \mathrm{M}\right)$ of barium chloride flowing at $2.4 \mathrm{~mL} \mathrm{~min}^{-1}$, showed linearity ranges from $5.0 \times 10^{-5}$ to $5.0 \times 10^{-3} \mathrm{M}$, with slopes of $76.4 \pm 0.6 \mathrm{mV} \mathrm{\text {decade } ^ { - 1 }}$ and $R^{2}>0.9935$. Slope decreased significantly under the requirement of a $\mathrm{pH}$ adjustment, selected at 4.5. Interference of several compounds (sodium, potassium, magnesium, barium, glucose, fructose, and sucrose) was estimated by potentiometric selectivity coefficients and considered negligible. Analysis of real samples were performed and considered accurate, with a relative error to an independent method of p2.7\%.

Key Words: Cysteine; Potentiometry; Ion-selective electrode; FIA and pharmaceuticals.

\section{INTRODUCTION}

Cysteine (Cys), the ( \pm )-2-Amino-3-mercaptopropionic acid, is an amino acid, classified as nonessential, since the human body can synthe- size it. Its external administration may be, however, required and is per- formed by means of commercialized pharmaceutical formulations. For human safety reasons, these formulations are subjected to several quality control requisites, among which the amount of main active compound is included. For this, the United States Pharmacopoeia (USP) suggests an iodimetric titration with sodium thiosulfate, having starch as endpoint indicator, and requiring a blank procedure for the necessary corrections. ${ }^{[1]}$ This method is somewhat elaborate, which makes it inadequate for the routine determinations required 
by industries. It involves a high number of manipulations by an operator, which may conduct to random errors as well as to unnecessary exposition to toxic compounds. Moreover, formu- lations concerned cannot present color, since it would conduct to an error at the end point estimation.

As a consequence, several other techniques dedicated to the determination of Cys in pharmaceutical formulations are described in literature, most of them implying optical techniques. Concerning UV/vis spectrophotometry, the reducing properties of Cys have been exploited allowing the establishment of indirect readings of the analyte. Concretely, $\mathrm{Fe}(\mathrm{III})$ is reduced to $\mathrm{Fe}$ (II) for formation of a colored complex with 1,10 phenanthroline ${ }^{[2]}$ or ferrozine. ${ }^{[3]}$ Other proposals are based in the chemical transformation of the analyte with $1,1^{0}$-thiocarbonyl- diimidazole ${ }^{[4]}$ or benzoquinone ${ }^{[5]}$ into an absorbing compound. The latter suffers from high interferences at the analysis of real matrices, for which a previous HPLC separation has been regarded by the authors. Fluorimetric procedures based in the ability of Cys to inhibit the fluores- cence of 8-hydroxyquinoline-5sulphonic acid and $\mathrm{Zn}(\mathrm{II})^{[6]}$ or $\mathrm{Cd}(\mathrm{II})^{[7]}$ complexes are proposed as well.

Other optical procedures employ the Flow Injection Analysis (FIA) technique, an adequate strategy for performing rapid and repetitive determinations; FIA has also distinct advantages in terms of cost, performance, and flexibility. The complexation of Co(II) and Cys, attained under flow and monitored at $360 \mathrm{~nm}$, is the basis of a proposal in Lit. ${ }^{[8]}$ The presented method has the particular feature of reducing consumption of Co by its entrapment into a solid phase reactor, which is an excellent strategy regarding economics and environment. Another flow spectrophotometric method is based as well in the redox properties of Cys, promoting a color change of Prussian blue to white. ${ }^{[9]}$ A low consumption of reagent is also here exploited, this time by means of an optical flow-through cell-detector with a transparent layer of Prussian blue incorporated.

In a general point of view, all above-mentioned optical methods enable the reading, at low cost, of low amounts of Cys after several sampleprocessing steps, established to eliminate interferences from color, turbidity, suspended solids, different refractive indexes, etc. Overall, chemical transformation or complexation of the analyte is here always regarded, which may, as a consequence, conduct to interference from excipients within the concerned matrices.

Without these features, a capillary electrophoresis based method connected to a reverse-pulse amperometric system with a gold-mercury amalgam microelectrode is reported in Lit. ${ }^{[10]}$ This method enables the elimination of interfering compounds, yet one main safety problem may arise from exposing the operator to mercury, a highly volatile and toxic compound. 
Other alternatives in literature regard electro-analytical techniques, usually less expensive than separative instrumental strategies. Among them, one voltammetric-based stationary procedure is able to electrocatalytically oxidize Cys by means of a redox ruthenium(III)/ diphenyldithiocarbamate mediator, applied to the surface of a carbon paste electrode. ${ }^{[11]}$ Biamperometric methods with two platinum electrodes are also reported, regarding a direct oxidation of $\mathrm{Cys}^{[12]}$ at a pretreated electrode or an indirect reading after reaction of analyte with chloramine$\mathrm{T}^{[13]}$ As a whole, techniques concerned present lack of selectivity, since every compound with redox properties may, under the applied potentials, give rise to analytical signals. This problem would be solved if the technique would be selective to Cys.

In fact, one Cys selective electrode, prepared by incorporating phthalocyanine into a plasticized PVC membrane, is reported. ${ }^{[14]}$ The resulting sensor shows Nernstian response and presents more than one-month lifetime. Although not a long lasting detection system, this potentiometric method does not required usual sample preparation steps. Nevertheless, it would be more adequate to a routine application if applied to flow conditions.

A FIA potentiometric research work, involving a selective electrode of $\mathrm{Ag} / \mathrm{Ag}_{2} \mathrm{~S}$, prepared by pretreatment of a silver tube, is indeed presented in Lit. ${ }^{[15]}$ The potentiometric response is sub-Nernstian and seems to be connected to the formation of an AgSR layer (the ionized thiol group of Cys complexes with silver), studied under batch conditions.

This electrode seems however not adequate for flow media due to the stream of carrier to which the membrane surface is subjected. Low lifetime detectors and a strong drift at analytical readings would be therefore expected. The formed complex could be propelled towards the waste, leading to a continuous leaching of sensor. The authors present another alternative, employing an $\mathrm{Ag} / \mathrm{AgI}$ selective electrode, equally prepared by pretreatment of a silver tube. The carrier presents iodine that, once reduced to iodide by Cys, gives rise to potential changes. This is of course an indirect measure, for which any reducing species among real matrices would contribute to analytical errors.

Considering all above-described methods, the construction of a Cys selective electrode of proper configuration for flow conditions would be of interest regarding the routine control of $\mathrm{Cys}$ required by industries. In this sense, selective electrodes will be proposed, constructed with a tubular arrangement. Since adequate working characteristics must be attained, several selective membranes will be established. Analysis of real samples will be attempted with the adequate membrane. 


\section{EXPERIMENTAL}

\section{Apparatus}

The potential differences between indicating and reference electrodes were measured by means of a Crison mpH 2002 decimilivoltammeter $( \pm$ $0.1 \mathrm{mV}$ sensitivity) coupled to a Metrohm E 586 recorder. The reference electrode was an Orion, 90-02-00, double junction electrode.

The selective electrode, with no internal reference solution, presented a

tubular configuration and was constructed as described elsewhere. ${ }^{[16]}$ When necessary, $\mathrm{pH}$ values were controlled by means of a Sentek 71728 combined glass electrode connected to a decimilivoltammeter.

The FIA system comprised a Gilson Minipuls 2 peristaltic pump, fitted with PVC tubing $(1.85 \mathrm{~mm}$ i.d.) and a four-way Rheodyne 5020 injection valve. All components were gathered by PTFE tubing (Omnifit, Teflon, 0.8 mm i.d.), Gilson end-fittings and connectors. The support devices for tubular and reference electrodes as well as ground electrode were constructed as reported elsewhere. ${ }^{[17]}$ 


\section{Reagents and Solutions}

All chemicals were of analytical grade and deionised water (conductivity $<0.1 \mathrm{mS} \mathrm{cm}^{-1}$ ) was used. L-cysteine hydrochloride monohydrate (Cys-HCl, Fluka), barium chloride (Fluka), o-phosphoric acid (85\%, Merck), sodium dihydrogenphosphate (Riedel-deHae" $\mathrm{n}$ ), and ammonia (Merck) were employed throughout. For the selective membrane preparation, bis(triphenylphosphoranyliden)ammonium (BTPPA) chloride (Aldrich), tetraphenylborate (TPB) sodium (Aldrich), bis(2ethylhexyl)sebacate ( $b$ EHS, Fluka), o-nitrophenyl octyl ether $(o$ NFOE, Fluka), poly(vinyl chloride) of high molecular weight (PVC, Fluka), and tetrahydrofuran (THF, Riedel-deHaen) were used. Evaluation of the effects of both $\mathrm{pH}$ and interfering ionic species at the potentiometric response required sodium hydroxide (Merck), hydrochloric acid (Merck), sodium chloride (Merck), potassium chloride (Merck), magnesium chloride (Riedel-de Hae" n), barium chloride (Merck), sucrose (Merck), glucose (Merck), and fructose (Merck).

Ionic strength (IS) was adjusted to $3 \times 10^{-3} \mathrm{M}$ by means of a $1 \times 10^{-3} \mathrm{M} \mathrm{BaCl} 2$ solution. Simultaneous $\mathrm{pH}$ and $\mathrm{IS}$ adjustment was

established with a $\mathrm{H}_{3} \mathrm{PO}_{4} / \mathrm{NaH}_{2} \mathrm{PO}_{4}$ buffer, prepared with different ratios of acid/base solutions in order to attain the intended $\mathrm{pH}$, and having always a final IS of $1 \times 10^{-2} \mathbf{M}$.

Standard solutions ranging $1.0 \times 10^{-5}$ to $5.0 \times 10^{-3} \mathrm{M}$ were prepared by accurate dilution of a stock Cys- $\mathrm{HCl}$ solution of $1.0 \times 10^{-2} \mathrm{M}$, all of these prepared in the carrier solution present at the concerned FIA trials (either IS or simultaneous $\mathrm{pH}$ and IS adjusters).

The $\mathrm{pH}$ influence was studied for a $5.0 \times 10^{-4} \mathrm{M}$ Cys- $\mathrm{HCl}$ solution prepared in IS adjuster. Interference from other chemicals was evaluated by the separated solutions method, ${ }^{[18]}$ for which $1.0 \times 10^{-4}, 5.0 \times 10^{-}$ 4 , and $1.0 \times 10^{-3} \mathrm{M}$ solutions of $\mathrm{Cys}-\mathrm{HCl}$ and sodium chloride, potassium

chloride, magnesium chloride, barium chloride, sucrose, glucose, or fructose, were prepared. These solutions were made in buffer $(4.5 \mathrm{pH}$ and 1 $\times 10^{-2}$ M IS). 


\section{Construction of Cys Selective Electrodes}

Ionic sensors were prepared by precipitation reaction after a mixture of $50 \mathrm{~mL}$ of a $1.0 \times 10^{-1} \mathrm{M}$ Cys- $\mathrm{HCl}$ solution and $50 \mathrm{~mL}$ of a $1.0 \times 10^{-2} \mathrm{M}$ sodium TPB or BTPPA chloride solution. Before reaction, Cys solution was added of a few drops of concentrated $\mathrm{HCl}$ or $\mathrm{NH} 3$ respectively, which increased the extent of the intended ionization and led to the formation of Cys-TPB or BTPPA-Cys ionic exchangers, respectively. The resulting precipitate was isolated from solution by filtration. After thorough washing with water, the filtered precipitate was kept in a dark flask inside a desiccator in order to prevent light and humidity alterations. Electrodes comprising the above ionic sensors will be further assigned as types $\mathrm{P}$ and $\mathrm{N}$, respectively.

Several sensor solutions were prepared by accurately dissolving approximately $0.02 \mathrm{~g}$ of the ionic sensor in $1.00 \mathrm{~g}$ of $o$ NFOE or $b$ EHS. The corresponding membrane solutions were prepared by mixing $0.20 \mathrm{~mL}$ of the previous solutions with $0.09 \mathrm{~g}$ of PVC, formerly dissolved in about $2 \mathrm{~mL}$ of THF. Membranes comprising those solvents will be after designated as types $\mathrm{O}$ and $\mathrm{B}$, respectively.

The selective electrodes were prepared by applying membrane solutions upon a tubular shape conductive support of graphite and epoxy resin. ${ }^{[16]}$ The membrane was let dry for $24 \mathrm{~h}$ and placed in contact

with a $1 \times 10^{-3} \mathrm{M}$ Cys- $\mathrm{HCl}$ solution prepared in water. When not in use, the electrodes were also kept under these conditions.

The resulting selective electrodes, whose membrane composition is indicated in Table 1, will be further addressed as types PO, PB, NO, and $\mathrm{NB}$, according to the sensor solutions they contain.

\section{Procedures}

The working characteristics of the selective electrodes were evaluated in a single-channel manifold having as carrier either IS or $\mathrm{pH}$ and IS adjuster solutions, an injection volume of $500 \mathrm{~mL}$ and a flow-rate of $2.4 \mathrm{~mL} \mathrm{~min}^{-1}$ (Fig. 1).

For studying the effect of $\mathrm{pH}$ at the potentiometric response, a volume of $200.0 \mathrm{~mL}$ of a Cys standard solution was made to circulate into a closed loop by the injection valve of the manifold depicted in 
Table 1. Membrane overall composition (\%,w/w) of the several Cys selective electrodes.

\begin{tabular}{lcccc}
\hline Components & PO & \multicolumn{1}{c}{ PB } & NO & NB \\
\hline Cys-TPB & 1.46 & 1.46 & - & - \\
BTFFA- & - & - & 1.4 & 1.34 \\
$o$ NFOE & 67.48 & - & 67.05 & - \\
$b$ EHS & - & 67.42 & - & 67.47 \\
PVC & 31.06 & 31.12 & 31.55 & 31.19 \\
\hline
\end{tabular}

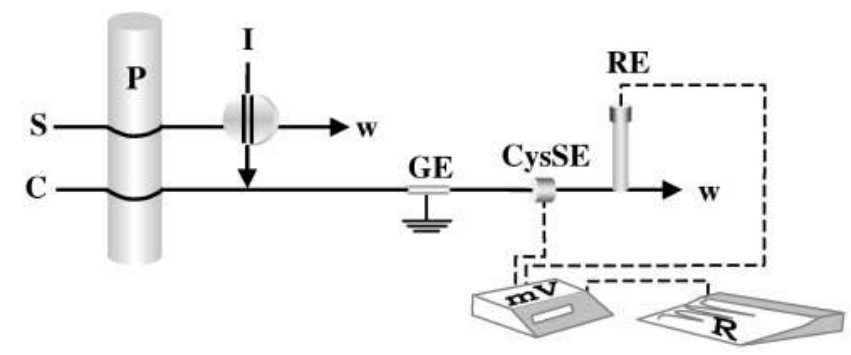

Figure 1. Flow injection manifold. P: peristaltic pump; S: sample; C: carrier $\left(\mathrm{BaCl}_{2}\right.$ for IS adjustment or phosphate buffer for both $\mathrm{pH}$ and IS adjustment); I: injection valve; GE: grounding electrode; CysSE: Cys selective electrode; RE: reference electrode; w: waste; $\mathrm{mV}$ : decimilivoltammeter; R: recorder.

Fig. 1. Alterations in $\mathrm{pH}$ of this solution were promoted by addition of small amounts of saturated $\mathrm{NaOH}$ and concentrated $\mathrm{HCl}$ solutions. These were selected regarding the nonintroduction of foreign chemical species.

The selectivity study was made by separately injecting $\mathrm{Cys}-\mathrm{HCl}$ and other possible interfering compound solutions into a carrier stream of phosphate buffer ( $\mathrm{pH} 4.5$ and IS of $1 \times 10^{-2} \mathrm{M}$ ).

The pharmaceutical preparation, commercialized as capsules, Phakan ${ }^{\circledR}$, contained a labeled amount of $290 \mathrm{mg}$ of Cys per unit, expressed in Cys-HCl. The average weight of each capsule was calculated in $470.4 \mathrm{mg}$. An exact amount of about $0.0128 \mathrm{~g}$ of powder was dissolved and after diluted with phosphate buffer $\left(\mathrm{pH} 4.5\right.$ and IS of $.1 \times 10^{-2} \mathrm{M}$ ) to a final volume of $50.00 \mathrm{~mL}$, leading to a stock solution of sample with a concentration estimated in $1.0 \times 10^{-3}$ M Cys. A $2500 \mathrm{~mL}$ volume of this solution was after diluted to a final volume of $25.00 \mathrm{~mL}$ with the same buffer; the expected concentration was of $1.0 \times 10^{-3} \mathrm{M}$ Cys. Recovery trials were preformed with a similar solution added of $1000 \mathrm{~mL}$ 
of a $1.00 \times 10^{-2} \mathrm{M}$ Cys standard solution before completing the final volume up to $25.00 \mathrm{~mL}$, equally with phosphate buffer.

Results of the potentiometric analyses were compared with those obtained with USP method. ${ }^{[1]}$ For this, an amount of sample containing $250 \mathrm{mg}$ Cys- $\mathrm{HCl}$ was dissolved in water and potassium iodide. This solution was cooled in an ice bath, added of $\mathrm{HCl}$ and iodine, and was let stand in the dark for $20 \mathrm{~min}$. Excess of iodine was titrated with thiosulphate sodium, having starch as end point indicator. A blank was required for corrections.

\section{RESULTS AND DISCUSSION}

In the construction of adequate working characteristics Cys selective electrodes, four different membranes were prepared. Considering that Cys presents amphoteric properties, the possibility of preparing both positively and negatively charged electrodes arose. For this, two distinct ionic exchangers were prepared, with Cys positively charged at the amine group (Cys-TPB) or negatively charged at the carboxylic group (BTPPA-Cys). Furthermore, membranes were prepared with either oNFOE or $b$ EHS. Besides different chemical structures, these solvents present quite opposite properties in terms of polarity: dielectric constants of 23.6 and 4.6, respectively. ${ }^{[19]}$

All prepared electrodes were evaluated under IS adjustment conditions, in order to select one for further applications.

\section{Working Characteristics of the Cys Selective Electrodes}

Working characteristics of the several Cys selective electrodes were established with the FIA manifold depicted in Fig. 1. A $500 \mathrm{~mL}$ injection volume and a $2.4 \mathrm{~mL} \mathrm{~min}^{-1}$ flow-rate were selected regarding the establishment of analytical conditions equivalent to those of stationary state.

Under IS adjustment conditions, positively charged electrodes gave a more or less predictable response (types PO and PB) while negative ones showed no logical behavior (types NO and NB) - instead of decreasing potential readings while concentration increased, only small positive peaks with random heights were attained. Considering that an alkaline medium would favor ionization of the carboxylic group, calibrations of types NO and NB detectors were tried again, this time with carrier and standard solutions having a $\mathrm{pH}$ of 10.9. Yet, no agreeing results were 
characteristics

Table 2. Working characteristics of types PO and PB Cys selective electrodes.

\begin{tabular}{lcc}
\hline \multirow{2}{*}{ Working } & \multicolumn{2}{c}{ IS $\left(3 \times 10^{-3} \mathrm{M}\right)$} \\
\cline { 2 - 3 } & $\mathrm{PO}$ & $\mathrm{PB}$ \\
\hline LLLR $(\mathrm{M})^{\mathrm{a}}$ & $5 \times 10^{-5}$ & $5 \times 10^{-5}$ \\
Slope $(\mathrm{mV}$ decade & $-1)$ & $54.6 \pm 0.1$ \\
Repeatability $( \pm \mathrm{Mv})^{\mathrm{b}}$ & $76.4 \pm 0.6$ & $\pm 0.6(4.1 \%)$ \\
$R^{2}$ & $\pm 1.1(1.1 \%)$ & $>0.9926$ \\
Sampling frequency $\left(\mathrm{h}^{-1}\right)$ & $>0.9935$ & 54 \\
\hline
\end{tabular}

a Lower limit of linear range.

${ }^{b}$ Average of 6-15 readings of Cys standard solutions.

attained. Opposing these results, types PO and PB electrodes showed good working characteristics (Table 2), with linearity ranges of 5 $\times 10^{-5}$ to $5 \times 10^{-3} \mathrm{M}$ Cys. High sensitivities were recorded for both electrodes, with emphasis to those of type PO. Completely unexpected, the latter presented slopes of $\mathbf{p} 74 \mathrm{mV}$ decade $^{-1}$; they had also better repeatability and a slightly quicker response than type PB detectors.

With regard to the intended analytical application, as well as to the $\mathrm{acid} / \mathrm{base}$ properties of Cys, the influence of the $\mathrm{pH}$ at the potentiometric response was evaluated. Cys presents three distinct $\mathrm{pK} a$ values, concerning the loss of $\mathrm{H}^{\mathrm{p}}$ by the carboxylic group (1.86), by the thiol group (8.35) and by the amine group (10.34). In this sense, the amount of positively charged Cys molecules in solution is straightly connected to its $\mathrm{pH}$. For a 2.0-11.0 $\mathrm{pH}$ range, both detectors showed an increase of analytical readings as $\mathrm{pH}$ decreased (Fig. 2). Probably, this behavior was not only connected to an increase of the positively charged Cys molecules, but also to the decrease of negative charges located at the same analyte. These opposite charges within the same chemical structure could have promoted an opposite effect at the expected analytical readings.

Though Reilley profiles were similar for both detectors, for an equal concentration of Cys peak heights were much smaller for type PB electrodes (Fig. 2), conducting to an apparent wide operational $\mathrm{pH}$ range. Indeed, peaks were here so small that it was difficult not to find constancy among recordings. These electrodes were therefore considered of no interest to the aimed analytical application and further trials proceeded with only type PO detectors. Being so, this distinct amphoteric property of Cys could not be fully exploited at this study, albeit other membrane compositions could allow the attainment of adequate potentiometric responses. 


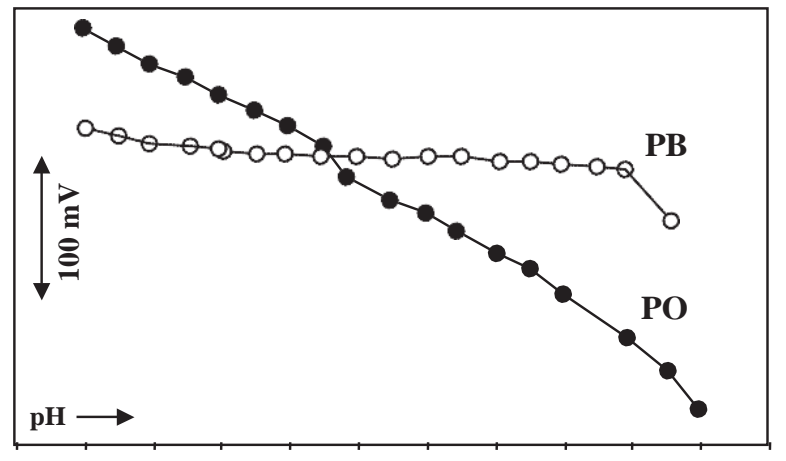

$\begin{array}{llllllllllll}1 & 2 & 3 & 4 & 5 & 6 & 7 & 8 & 9 & 10 & 11 & 12\end{array}$

Figure 2. Reilley diagrams of types $\mathrm{PO}$ and $\mathrm{PB}$ electrodes for a $\mathrm{Cys}-\mathrm{HCl}$ solution of $5 \times 10^{-4} \mathrm{M}$ in IS adjuster.

Regarding type PO electrodes, the Reilley diagram showed no operational $\mathrm{pH}$ range (Fig. 2), with a continuous potential change as $\mathrm{pH}$ drifted to lower values. This lack of constancy resulted most probably from the acid/base properties of the concerned analyte. In this sense, several calibrations were attempted at different acidic pHs. Results attained here pointed out an increasing interference from $\mathrm{H}^{\mathrm{p}}$ as $\mathrm{pH}$ decreased, since quite small sensitivities as well as unstable potentials were recorded; when $\mathrm{pH}$ was increased, nonlinear results were attained due to a significant raise at the lower limit of linear range. Acceptable recordings ranged only 4-5 pH units.

Best behavior under $\mathrm{pH}$ adjustment conditions was recorded for a $4.5 \mathrm{pH}$ (Table 3), though the slope was sub-Nernstenian, and quite distinct from the one attained under IS adjustment (Table 2). This slope variation may regard the formation of different ratio complexes within the selective membrane, though no information in literature confirms this possibility. Moreover, air oxidation of thiol groups of two Cys molecules (forming cystine, the disulfide-linked dimmer of Cys), could have reduced the concentration of protonated Cys in solution. ${ }^{[20]}$ Reductive cleavage could be accomplished by reaction with b-mercaptoethanol, converting cystine functional groups back into Cys. ${ }^{[20]}$ This was tried out for a $1 \times 10^{-4}$ $\mathbf{M}$ concentration of reducing compound, at both carrier and standard solutions. Results did not improve significantly and a higher concentration of b-mercaptoethanol was not recommended due to its high impact at the environment.

Interference of other compounds regarding Cys determinations was also evaluated under the previously selected $\mathrm{pH}$ conditions. 
Table 3. Working characteristics of type $\mathrm{PO}$ detector at several $\mathrm{pH}$ values.

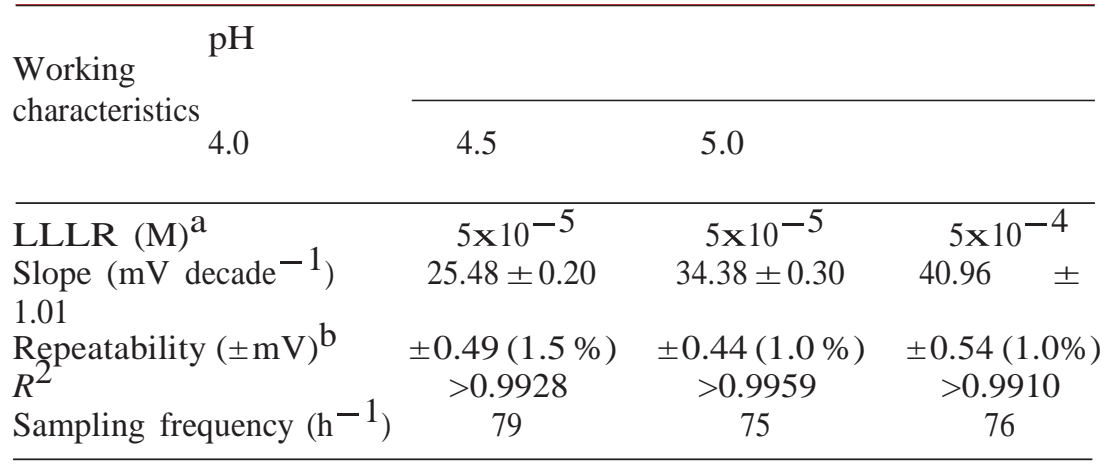

${ }^{\mathrm{a}}$ Lower limit of linear range.

${ }^{b}$ Average of 6-15 readings of Cys standard solutions.

Table 4. Potentiometric selectivity coefficients of type $\mathrm{PO}_{4} \mathrm{Cys}$ selective electrodes for concentrations of $1.0 \times 10^{-4}$ (A), $5.0 \times 10^{-4}$ (B), and $1.0 \times 10^{-3}$

(C) $\mathrm{M}$.

\begin{tabular}{lccc}
\hline Interference & A & B & C \\
\hline Sodium & $-0.363 \pm 0.008$ & $-1.062 \pm 0.013$ & -1.315 \\
0.006 & & & \\
Potassium & $-0.172 \pm 0.001$ & $-0.879 \pm 0.002$ & $-1.157 \pm 0.001$ \\
Magnesium & $-2.160 \pm 0.006$ & $-2.477 \pm 0.012$ & $-2.551 \pm 0.003$ \\
Barium $_{\text {Fructose }^{\mathrm{a}}}$ & $-2.413 \pm 0.020$ & $-2.714 \pm 0.007$ & $-2.780 \pm 0.004$ \\
Sucrose $^{\mathrm{a}}$ & $-2.477 \pm 0.021$ & $-2.824 \pm 0.034$ & $-2.978 \pm 0.017$ \\
Glucose $^{\mathrm{a}}$ & $-2.480 \pm 0.003$ & $-2.819 \pm 0.001$ & $-2.929 \pm 0.013$ \\
& $-2.182 \pm 0.003$ & $-2.542 \pm 0.010$ & $-2.670 \pm 0.009$
\end{tabular}

a Assuming ionization of a p1 charge.

Potentiometric selectivity coefficients were calculated for several concentrations and for several compounds, as indicated in Table 4. Basically, interference of inorganic cations was considered negligible. Lower coefficients for doubly charged ions were recorded and potassium showed a higher effect than sodium due to its volume size, more proximal to an ammonium ion. Among organic compounds, only carbohydrates were tested. Since they were not positively ionized, and in order to assume its higher interference possible (theoretically), they were assumed as singly charged cations. Results were similar for all carbohydrates, and indicated a negligible interference as well. All calculated selectivity coefficients, which can only be assumed as mere indication of the electro- de's behavior when in contact with coexisting ions, suggest that Cys selective electrode will behave accurately at the analysis of real matrices. 
It is important to state that results here were attained at the same $\mathrm{pH}$ and IS values that are to be used at the intended analytical application.

\section{Optimization of the FIA Set-Up}

Optimization was accomplished by checking one parameter and keeping others constant, regarding $100-1000 \mathrm{~mL}$ sampling volumes and 2.0-10.0 $\mathrm{mL} \mathrm{min}^{-1}$ flow rates. Recordings from previous conditions were mainly evaluated in terms of dispersion ${ }^{[21]}$ and sampling rates. Whilst injection of $200 \mathrm{~mL}$ gave rise to high dispersion (3.3), $500 \mathrm{~mL}$ ensured the necessary low dispersion (1.2). Though nondispersion was only attained with $1000 \mathrm{~mL}$, this condition was coupled to a $47 \%$ decrease in sampling rate if compared to results of $500 \mathrm{~mL}$. Thus, the latter was selected for further trials. The flow rate increase after $4 \mathrm{~mL} \mathrm{~min}^{-1}$ conducted to a significant increase in dispersion, up to a maximum of three units. For a more or less constant dispersion ranging $2.6-3.4 \mathrm{~mL} \mathrm{~min}^{-1}$, the lowest flow-rate was selected. Due to the fact that sampling-rates did not vary significantly within this range (around 90 to 100 samples $\mathrm{h}^{-1}$ ), the reagent consumption and environment where here a main concern.

\section{Application to Real Samples}

Cys was potentiometrically determined in the only commercialized pharmaceutical preparation after calibrating the FIA system under fixed $\mathrm{pH}$ and IS (Fig. 3). Before injection, every sample was diluted in order to fit the calibration curve. The results obtained are indicated in Table 5, and correspond to average and standard deviation of eight determinations. The resulting FIA records indicated that the proposed system was able to analyze about 81 diluted samples $\mathrm{h}^{-1}$.

Accuracy of potentiometric results was ascertained by relative deviations lower than 3\%, when compared to the USP method (Table 5). Recovery trials were also performed, and confirmed the accuracy of the potentiometric method. Additionally, considering as null hypothesis that variances of the two methods are in agreement, $F$ two tail test for a $5 \%$ level of significance gave a calculated $F(22.12)$ below the critical $F$-value $(F 0.025(2,7) 1 / 439.36)$, therefore accepting the null hypothesis.

Regarding reagent consumption in the analysis of real samples, calibration of the proposed method required about $30 \mathrm{~mL}$ of phosphate buffer and $1.5 \mathrm{~mL}$ of each standard solution, with concentrations ranging $5 \times 10^{-5}-5 \times 10^{-3} \mathrm{M}$ (considering three injections per standard). 


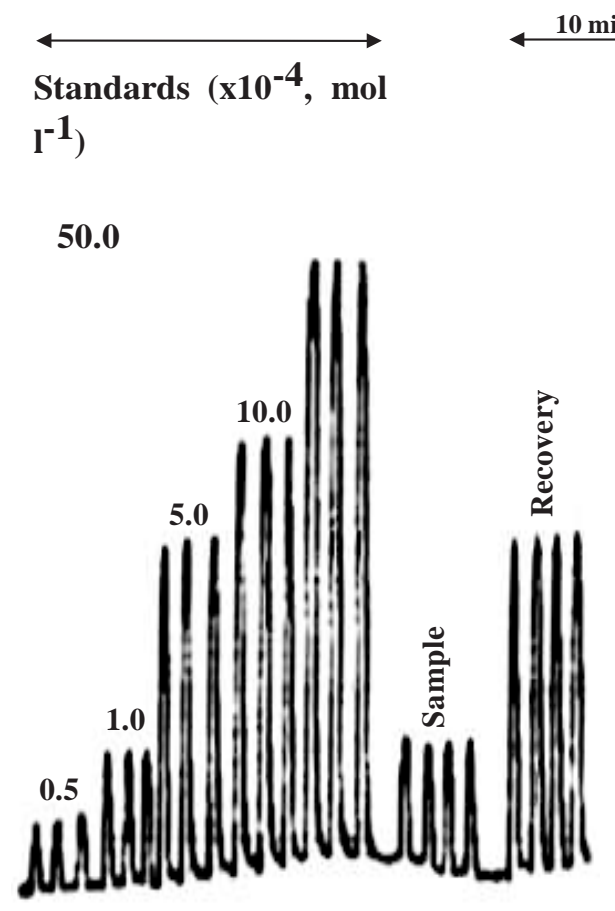

Figure 3. FIA output for a diluted pharmaceutical sample and its recovery after calibration.

Table 5. Determination of Cys by the potentiometric FIA method (POT), its recovery, and by the comparison method (USP), as well as the corresponding relative error (RE) and the calculated $F$ value.

\begin{tabular}{|c|c|c|c|c|c|c|}
\hline \multirow[t]{2}{*}{ 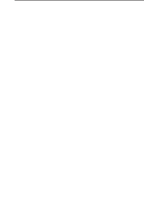 } & 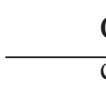 & $\frac{\text { Cys-HCl }}{\text { capsule }_{1}}$ & $(\mathrm{mg}$ & \multirow[b]{2}{*}{$\begin{array}{l}\text { Recover } \\
\mathrm{y} \\
(\%)\end{array}$} & \multirow[b]{2}{*}{$\begin{array}{l}\mathrm{RE} \\
(\%)\end{array}$} & \multirow[b]{2}{*}{$F$ test } \\
\hline & labeled & $\begin{array}{l}\text { POT } \\
\text { USP }\end{array}$ & & & & \\
\hline Capsule & 290 & $256.15 \pm 6.35$ & $249.38 \pm 2.35$ & $99.8 \pm 1.8$ & p2.71 & 22.12 \\
\hline
\end{tabular}

Moreover, several FIA determinations demonstrated the possibility of analyzing four different diluted samples without requiring re-calibration. Effect to the environment of the emitted effluents was, generally, considered of no concern, both due to its low volume and to its com- position (phosphate and Cys). An adjustment of $\mathrm{pH}$ before discard is most certainly required, and the intensity of further pollution depends on the receptor; phosphate may be of interest to agriculture. 


\section{CONCLUSIONS}

Regarding type PO membrane, Cys determination in pharmaceutical preparations using potentiometric detection presents a good alternative to both USP and other methods previously described in literature. The proposed system is simple, accurate, inexpensive regarding reagent consumption and equipment involved, and not of concern in terms of emitted effluents, for which it is adequate for routine procedures. Aside from dissolution and dilution, no sample preparation steps are required. Lifetime

of the detector is estimated for at least four months; longer periods were not tested.

\section{REFERENCES}

1. The United States Pharmacopoeia 24, the National Formulary 19, Official monograph for Cysteine hydrochloride, 2000, 490.

2. Teshima, N.; Nobuta, T.; Sakai, T.; Kawashima, T. Utilization of a copper catalysis for the spectrophotometric determination of cysteine. Bunseki Kagaku 2001, 50 (1), 47-51.

3. Abu-Eid, M. Spectrophotometric determination of cysteine and $\mathrm{N}$ acetylcysteine in pharmaceutical preparations. Mikrochim. Acta 1998, $129(1-2), 91-95$.

4. Amarnath, V.; Amarnath, K. Specific determination of cysteine and penicillamine through cyclization to 2-thioxothiazolidine-4- carboxylic acids. Talanta 2002, 56 (4), 745-751.

5. Zaia, D.A.M.; Ribas, K.C.L.; Zaia, C.T.B.V. Spectrophotometric determination of cysteine and/or carbocysteine in a mixture of amino acids, shampoo, and pharmaceutical products using $p$-benzoquinone. Talanta 1999, 50 (5), 1003-1010.

6. Wang, H.; Wang, W.S.; Zhang, H.S. A spectrofluorimetric method for cysteine and glutathione using the fluorescence system of $\mathrm{Zn}(\mathrm{II})-8$ hydroxyquinoline-5-sulphonic acid complex. Spectroc. Acta Part A 2001, 57 (12), 2403-2407.

7. Wang, H.; Wang, W.S.; Zhang, H.S. Spectrofluorimetic determina-tion of cysteine based on the fluorescence inhibition of $\mathrm{Cd}(\mathrm{II})-8$ hydroxyquinoline-5-sulphonic acid complex by cysteine. Talanta 2001, 53 (5), 1015-1019.

8. Icardo, M.C.; Zamora, L.L.; Calatayud, J.M. Solid-phase reactors as high stability reagent sources in flow analysis: selective flow injection spectrophotometric determination of cysteine in pharmaceutical formulations. Analyst 1998, 123 (8), 1685-1689. 
9. Lenarczuk, T.; Glab, S.; Koncki, R. Application of Prussian bluebased optical sensor in pharmaceutical analysis. J. Pharm. Biomed. Anal. 2001, 26 (1), 163-169.

10. Jin, W.R.; Xu, Q.; Li, W. A new capillary electrophoresis endcolumn amperometric detection system without the need for capillary/electrode alignment. Electrophoresis 2000, 21 (8), 15271534.

11. Nalini, B.; Narayanan, S.S. Electrocatalytic oxidation of sulfhydryl compounds at ruthenium(III) diphenyldithiocarbamate modified carbon paste electrode. Electroanalysis 1998, 10 (11), 779-783.

12. Zhao, C.; Zhang, J.C.; Song, J.F. Determination of L-cysteine in amino acid mixture and human urine by flow-injection analysis with a biamperometric detector. Anal. Biochem. 2001, 297 (2), 170176.

13. Icardo, M.C.; Estrela, O.A.; Sajewicz, M.; Mateo, J.V.G; Calatayud, J.M. Selective flow-injection biamperometric determination of sulfur-containing amino acids and structurally related compounds. Anal. Chim. Acta 2001, 438 (1-2), 281-289.

14. Shahrokhian, S. Lead phthalocyanine as a selective carrier for preparation of a cysteine-selective electrode. Anal. Chem. 2001, 73 (24), 5972-5978.

15. Kolar, M.; Dobcnik, D.; Radic, N. Chemically treated silver electrodes for the determination of cysteine. Mikrochim. Acta 2002, $138(1-2), 23-27$.

16. Alegret, S.; Alonso, J.; Bartroli, J.; Paulis, J.M.; Lima, J.L.F.C.; Machado, A.A.S.C. Flow-through tubular PVC matrix membraneelectrode without inner reference solution for flow- injection analysis. Anal. Chim. Acta 1984, 164 (Oct.), 147-152.

17. Alegret, S.; Alonso, J.; Bartroli, J.; Machado, A.A.S.C.; Lima, J.L.F.C.; Paulis, J.M. Construction of equipment for potentiometric determinations in flow-injection analysis. Quim. Anal. 1987, 6(1), 278-294.

18. Buck, R.P.; Linder, E. International Union of Pure and Applied Chemistry (IUPAC), Analytical Chemistry Division Commission on Electroanalytical Chemistry, recommendations for nomenclature of ion-selective electrodes. Pure Appl. Chem. 1994, 66 (12), 2527-2536.

19. Okada, T.; Sugihara, H.; Hiratani, K. Role of plasticizers on the characteristics of poly(vinyl chloride)-membrane lithium-selective electrodes based on phenanthroline derivatives. Analyst 1995, 120 (9), 2381-2386. 
20. Schmid, G.H. Organic Chemistry; Black, L.W., Ed.; Mosby-Year Book: Toronto, 1996; 1154.

21. Trojanowicz, M.; Matuszewski, W. Limitation of linear response in flow-injection systems with ion-selective electrodes. Anal. Chim. Acta 1982, 138 (Jun.), 71-79. 